\title{
Post-operative computed tomography imaging evaluation of ascending aorta surgery
}

\author{
Patrizia Toia ${ }^{1 \mathrm{E}}$, Luciano Pennisi ${ }^{1 \mathrm{E}, \mathrm{F}}$, Rossana Taravella ${ }^{1 \mathrm{E}, \mathrm{F}}$, Emanuele Grassedonio ${ }^{1 \mathrm{E}}$, Cesare Gagliardo ${ }^{1 \mathrm{E}}$, \\ Massimo Galia ${ }^{1 \mathrm{E}}$, Massimo Midiri" ${ }^{1 \mathrm{E}}$, Ludovico La Grutta ${ }^{2 \mathrm{E}}$ \\ 'Department of Biomedicine, Neurosciences and Advanced Diagnostics - Bi.N.D., University of Palermo, Palermo, Italy \\ ${ }^{2}$ Department of Health Promotion, Mother and Child Care, Internal Medicine and Medical Specialties - ProMISE, University of Palermo, Palermo, Italy
}

\begin{abstract}
Ascending thoracic aorta disease is often a life-threatening condition. Aortic aneurysm and aortic dissection are the most frequent ascending aorta diseases requiring surgical intervention. Surgical repair techniques of the ascending aorta are various; they include reconstruction of the ascending aorta by using a graft with or without a prosthetic valve, reconstruction with a composite artificial graft or using a biological graft, and reconstruction of the ascending aorta with a composite graft preserving the native valve and arch repair. The radiologist plays a key role in the identification of post-operative complications; differentiation from normal postoperative findings is fundamental. Our aim is to discuss the main diseases affecting the ascending aorta requiring surgery and the different techniques used to treat them. We also discuss the normal computed tomography (CT) imaging findings and after-surgery complications.
\end{abstract}

Key words: post-operative CT imaging, post-operative complications, ascending aorta-surgery, graft, Bentall-De Bono procedure, elephant-trunk procedure.

\section{Introduction}

Pathologies of the ascending tract of the thoracic aorta are often life-threatening.

A multitude of surgical techniques are used to repair damaged aorta, such as the Wheat procedure (consisting of placing an aortic graft and an aortic valve prosthesis), Bentall and Cabrol approaches (by placing a composite artificial graft), Ross intervention (implanting a biological aortic graft), David technique and its derivatives (allocating an aortic graft without any intervention on the aortic valve), and elephant trunk (arch repair).

A proper imaging computed tomography (CT) protocol facilitates a correct diagnosis.

In this article, we discuss the main diseases affecting the ascending aorta and the different surgical techniques used to treat them. We also discuss the computed tomo- graphy imaging findings before and after surgery, including some of the most common complications.

\section{Discussion}

\section{Pathological conditions}

\section{Aortic aneurysm}

Aneurysm of the aorta is a focal and persistent enlargement of all aortic wall layers greater than $50 \%$ compared to a normal calibre [1].

The thoracic aorta is made up of the aortic root, ascending tract, aortic arch, and descending section. The ascending segment spans from the root to the origin of the right brachiocephalic artery; the arch, from the right brachiocephalic artery to the attachment of the ligamentum arteriosum; and the descending section, from the ligamentum

\section{Correspondence address:}

Patrizia Toia, Department of Biomedicine, Neurosciences and Advanced Diagnostics - Bi.N.D., University of Palermo, Via del Vespro 127, 90100, Palermo, Italy,

e-mail: toiapatrizia@gmail.com 
arteriosum to the aortic hiatus of the diaphragm. The aortic root comprises the valve, annulus and sinuses [2].

A frequent cause of aneurysm of the ascending segment is caused by cystic medial degeneration; in this condition, looking at histology samples, muscle cells are less represented and loss of function of the elastic fibres is noted.

This process leads to aneurysm of the vessel, by strength loss of the wall layers [3].

Clinical studies describe vastly different growth rates of the aneurysms of the ascending tract of the aorta spanning from $0.07 \mathrm{~cm} / \mathrm{y}$ to $0.42 \mathrm{~cm} / \mathrm{y}$. As the dilatation grows, so does the risk of life-threatening events; in patients with a $6 \mathrm{~cm}$ aneurysm, risk of rupture, dissection or death reaches $14.1 \%$ [4]. In some associated diseases, such as Marfan [4], Loeys-Dietz syndrome [5], and abnormal congenital aortic valve (such as bicuspid valve), surgery should be performed earlier. In particular, a diameter of $4.5 \mathrm{~cm}$ or greater is considered as a cut-off for surgical operation [6].

Other causes of aortic enlargement and aneurysms are large vessel vasculitis, for example Takayasu arteritis [7].

\section{Aortic dissection}

The aortic wall is made up of 3 stratums (from the innermost to the outermost): intima, media, and adventitia.

Longitudinal laceration of the aortic wall, allowing blood to flow between the intima and the adventitia, dividing the media layer, is called dissection [8]. In this condition 2 lumina are created, a false and a true lumen [9].

Because of the superior pressure inside the false lumen, the true lumen is smaller and may be compressed or obstructed. The false lumen may remain patent, close due to thrombosis, unite with the true lumen distally, or rupture, allowing blood to spill into nearby structures such as the pericardial, pleural, or peritoneal cavities. If the dissection extends to side branch arteries, such as cerebral, coronary, mesenteric, or renal, ischaemia is a probable occurrence due to reduced blood flow to these districts [10].

Aortic dissection is currently classified using the Stanford classification, which includes 2 types based on the required surgical intervention: $\mathrm{A}$ and $\mathrm{B}$. The former is the most frequent, accounting for $75 \%$ of cases [11]; in type A, dissection involves the ascending aorta or both the ascending and aortic arch or also descending aorta; acute type A dissection represents an emergency condition due to potentially fatal complications, such as involvement of the coronary arteries, the aortic valve, or rupture into chest cavities such as the pleural space or the pericardium (hence cardiac tamponade), warranting prompt surgical intervention.

Chronic type A dissection is also treated surgically; often, in this condition, medial layer disease is present (such as cystic medial necrosis).

Stanford type B is dissection of the aorta originating distal to the left subclavian artery. Usually this type does not require surgical intervention, and medical treatment is the option of choice unless signs of progression of the disease arise (for example, persistent abdominal pain, suggestive, in the absence of other causes, of bowel ischaemia) [11].

\section{Surgical approaches and computed tomography imaging findings}

Throughout the years different surgical repair techniques have been invented. Some examples are reconstruction of the ascending aorta by using a graft with (Wheat procedure) or without a prosthetic valve; reconstruction with a composite artificial graft (Bentall and Cabrol approaches), or placing a biological graft (Ross intervention and homograft reconstruction); rebuilding of the ascending aorta with a composite graft, retaining the patient's valve (David technique and derivatives); arch repair (elephant trunk intervention).

In almost all surgical techniques the patient's damaged aorta is removed and a graft (whether biological or artificial) is implanted. Nowadays the graft is connected to the patient's undamaged vessel, because it always gives better results than the previous method, where the graft was positioned within the damaged aorta - a technique called inclusion graft [12].

Synthetic grafts are frequently made of polyethylene (Dacron). At baseline CT, they have slightly higher density than the native aortic wall [13].

After administration of intravenous contrast material, grafts may not be identifiable on the CT images, due to similar attenuation values with the contrast agent. The felt ring, instead, is slightly denser because of its thickness, and is thus recognisable. It is crucial to identify these structures because they may mimic pathologic conditions, such as pseudoaneurysms. For this reason, acquisition of both baseline and angiographic phase CT are advised in these patients [13] (Figure 1). An ECG-gated protocol allows the reduction of motion artifacts together with the evaluation of coronary arteries.

After surgery the usual imaging findings may consist of perigraft fluid collections and mediastinal air; fluid collections do not show any enhancement on contrast CT and are reabsorbed over time, not prompting intervention is required [14] (Figure 2).

However, if contrast enhancement is noted, or fluid collection increases in volume on the follow-up CT, an infection should be suspected. Moreover, postoperative mediastinal air is another usual finding [13]; persistent or new perigraft air raises the suspicion of anaerobic bacterial infection or formation of a fistula with the adjacent structures such as bronchus or oesophagus [14].

\section{Coronary ostia sparing grafts}

In 1964, Wheat and his pears performed successfully an intervention in which an aneurismatic ascending aorta was excised, the coronary ostia with a small part of the annulus retained, and the patient's aortic valve was concurrently 

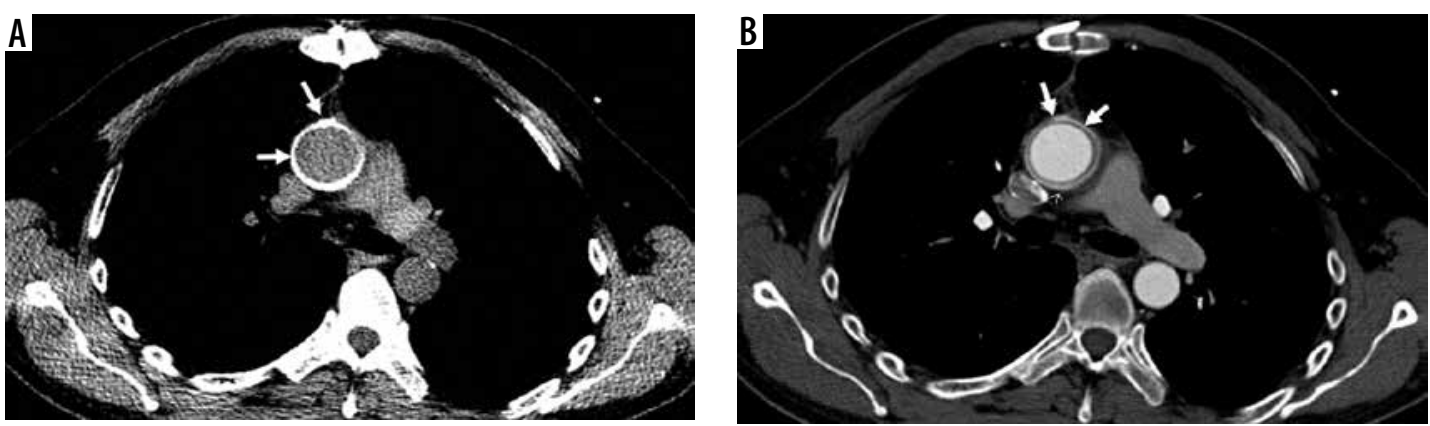

Figure 1. Aortic graft in the ascending aorta. Computed tomography scan (axial view) of an aortic graft in the ascending aorta: A) before contrast administration (best delineating the well-allocated graft, arrows) and B) after contrast administration (the graft is less distinguishable, arrows)
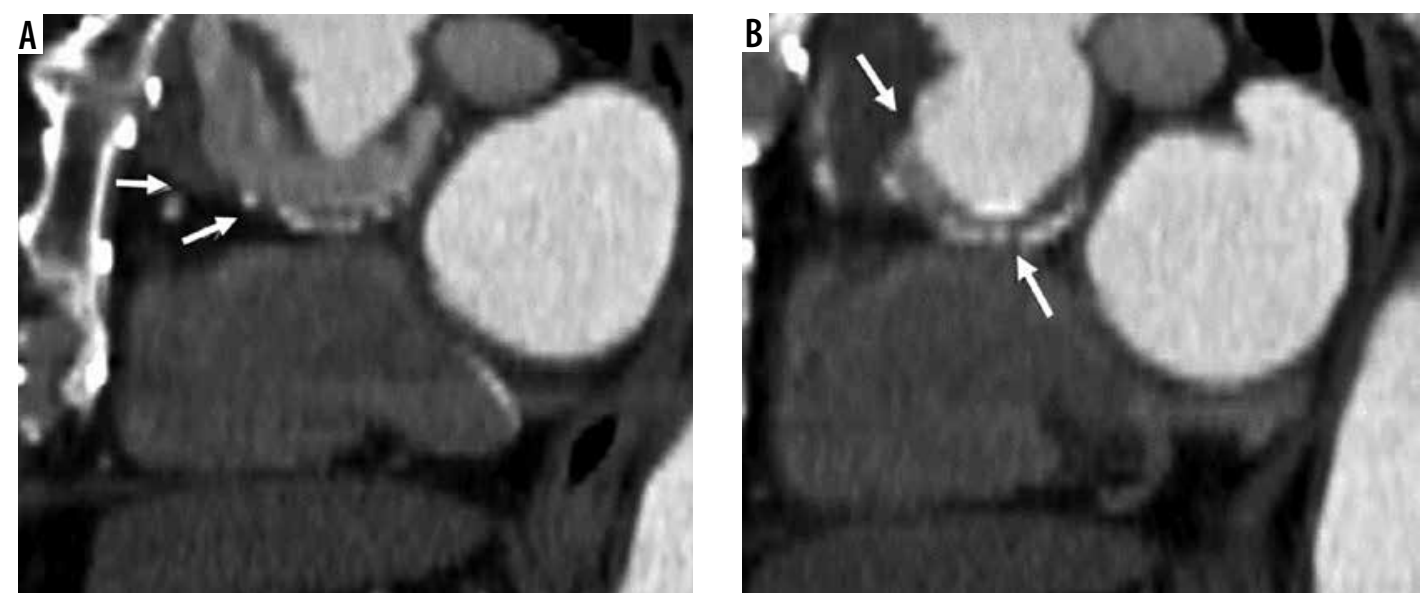

Figure 2. Perigraft fluid. Sagittal view, post-contrast computed tomography scan showing perigraft fluid (A and B, arrows)
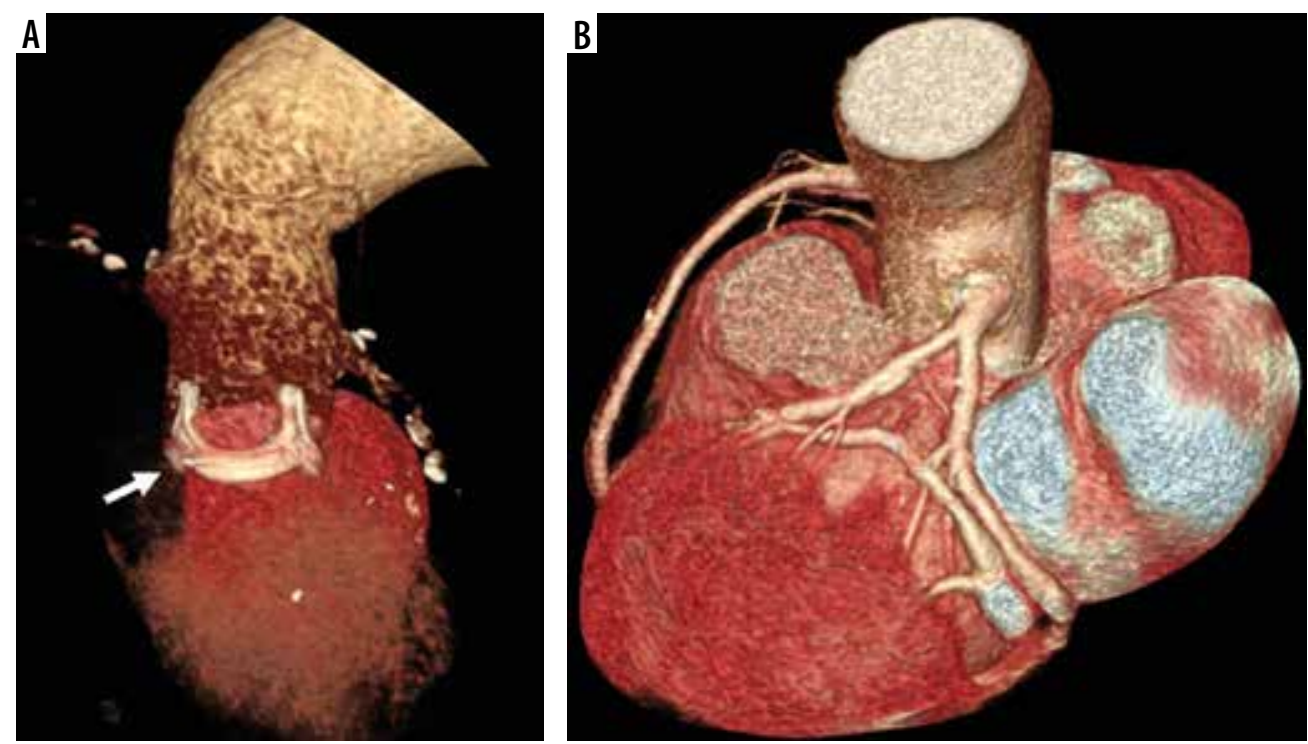

Figure 3. Bentall procedure. 3D volume rendering computed tomography images show Bentall procedure with a prosthetic valve implantation ( $A$, arrow) and anastomoses of the coronary ostia to the graft (B)

substituted. Since then this approach has been known as the Wheat procedure [15].

Sparing of the coronary ostia (that are anastomosed with the graft) reduces the risk of thrombosis, stenosis, kinking, and pseudoaneurysms at the coronary anastomosis spot [15].

One possible complication of this technique is dissection or aneurysm of the native aorta proximal to the graft. Some comorbidities increase the risk of these events, such as patients with annuloaortic ectasia or Marfan syndrome [12].

\section{Composite and biological grafts}

In 1968, Bentall and De Bono [16] conceived a procedure in patients with enlargement of both the ascending aorta and 

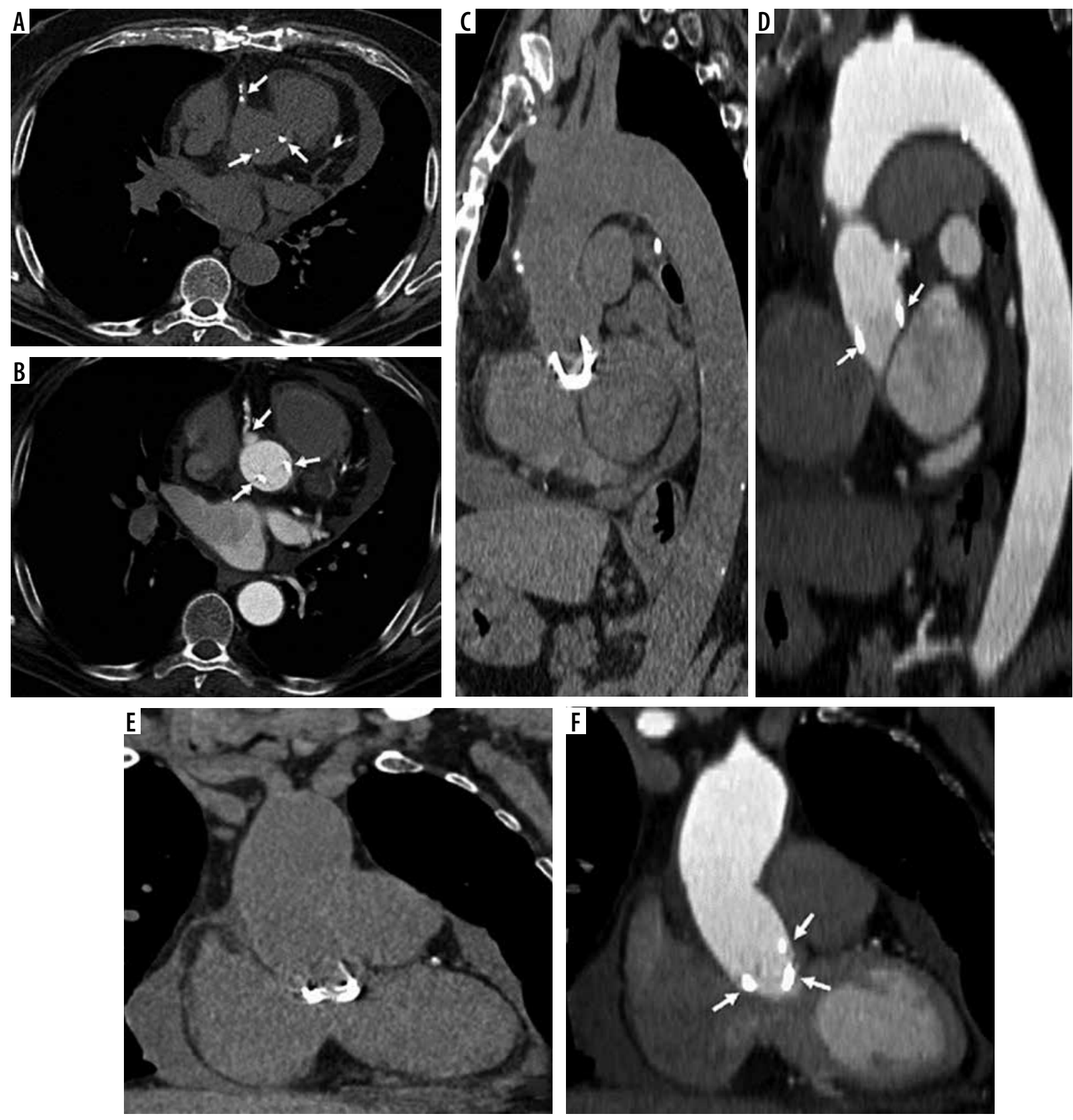

Figure 4. Modified Bentall procedure. Computed tomography (CT) scan showing a patient with modified Bentall procedure with pericardial effusion ( A - axial, C - sagittal, E - coronal CT scans, before and B - axial, D - sagittal, F - coronal, after intravenous contrast administration)

the aortic root. In this technique, the ascending aorta and the aortic root are removed and replaced by a graft. The coronary ostia are attached to the graft, and the patient's aortic valve is taken out and replaced by a prosthetic one (Figure 3 ).

Frequent complications at the site of the coronary ostia attachments (kinking and thrombosis) warranted a new approach, known as "modified Bentall" or the "button Bentall" or "Carrel patch". In this intervention, a round portion of the aorta surrounding the coronary ostia (the "button") and the coronary arteries are excised. This allows easier reimplantation of the of coronary arteries to the graft [17] and a meaningful drop in complications, as mentioned in a retrospective study by Milano et al., in which out of 71 patients only $4(6 \%)$ encountered complications [18] (Figure 4).
These techniques, often performed by surgeons, present some drawbacks, such as the formation of pseudoaneurysms distal to the anastomoses site and dissection (Figures 5 and 6).

In 1981, another technique was developed - the Cabrol procedure - in order to offer an alternative approach to patients with aortic dissection or annuloaortic ectasia [12]. In the Cabrol technique, the native aortic valve and ascending aorta are removed and a new valve and a synthetic graft are placed; the coronary ostia are attached to prosthetic conduits and the latter are then connected to the aortic graft [19].

Some complications consist of anastomotic stenosis, periprosthetic hematoma and pseudoaneurysm $[19,20]$.

Modified Bentall procedure is generally preferred [20]. 

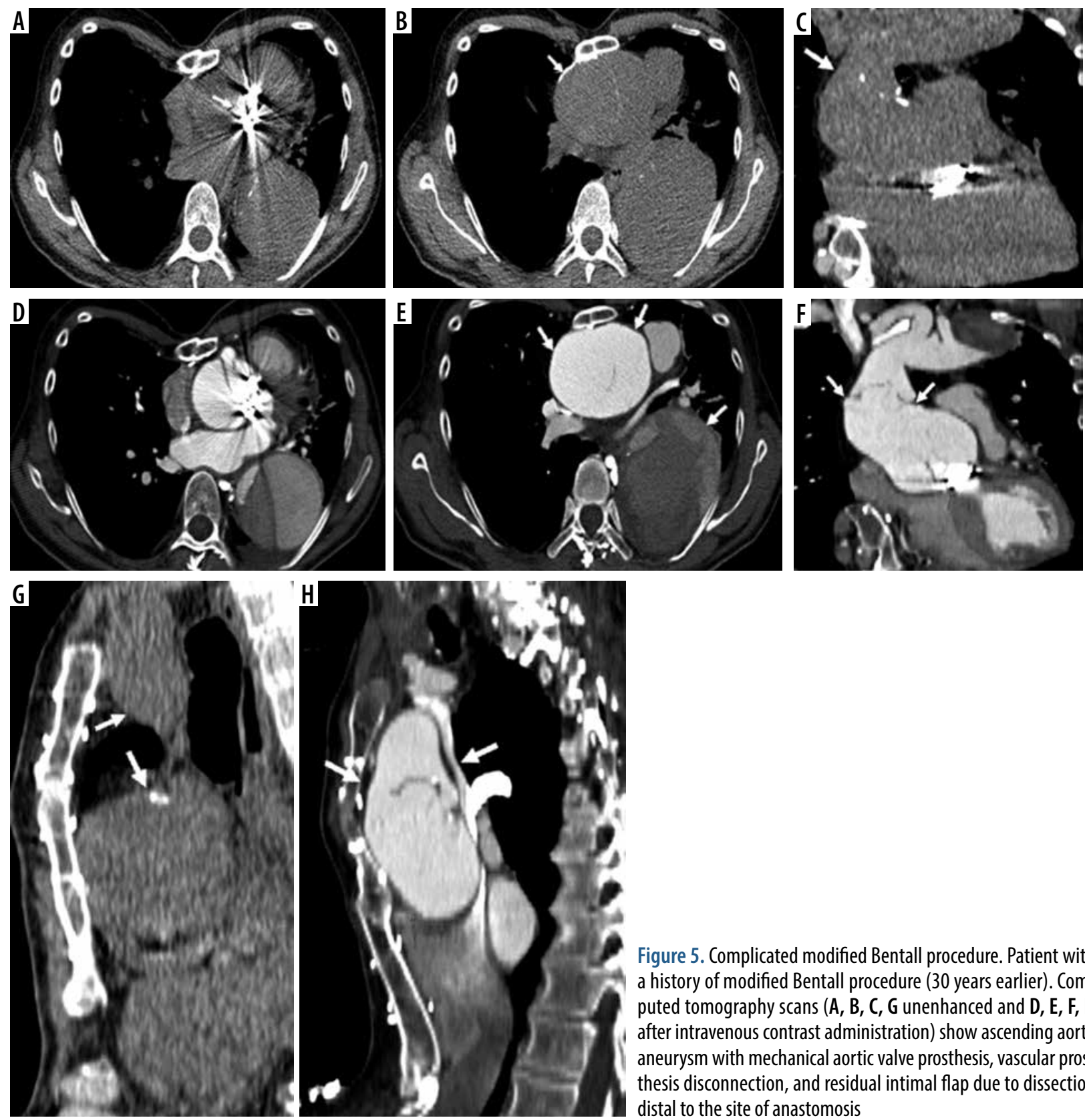

Figure 5. Complicated modified Bentall procedure. Patient with a history of modified Bentall procedure (30 years earlier). Computed tomography scans (A, B, C, G unenhanced and D, E, F, H after intravenous contrast administration) show ascending aorta aneurysm with mechanical aortic valve prosthesis, vascular prosthesis disconnection, and residual intimal flap due to dissection distal to the site of anastomosis

Biological grafts are instead often used in young patients; in the Ross intervention, the patient's pulmonary root replaces the native aortic root and an allograft is used to connect the right ventricle to the pulmonary artery [21], the main advantage is that anticoagulant therapy is not needed. The disadvantages include the duration of the valve (needing replacement after 10-20 years), complexity of the surgery, and potential complications to both valves (pulmonary and aortic) [21] and pseudoaneurysms located at both attachment sites [22].

\section{Aortic valve-sparing techniques}

Since artificial aortic valves require life-long anticoagulation therapy, other surgical repair procedures have been developed in order to preserve the native aortic valve while repairing a damaged ascending aorta [23]. Two main exam- ples of valve-sparing aortic root repair (V-SARR) include the remodelling technique by Yacoub and the reimplantation procedure by David $[24,25]$.

The former consists of asportation of the aneurysmatic aorta to the annulus level, keeping the native aortic valve in place and reconstructing the sinuses of Valsalva by inserting an artificial graft [24].

In the David technique the ascending aorta is removed, a graft is attached below the native valve, and the latter is introduced inside the graft [25]. In both techniques the coronary arteries are reimplanted into the aortic graft (Figure 7).

Some modifications of these technique have been invented, such as the T. David-V technique; in this procedure, the sinuses of Valsalva are reconstructed using a larger artificial graft. In the Demers and Miller intervention, 2 aortic grafts are connected together to create new sinuses; these are even wider than the ones of the $\mathrm{T}$. 

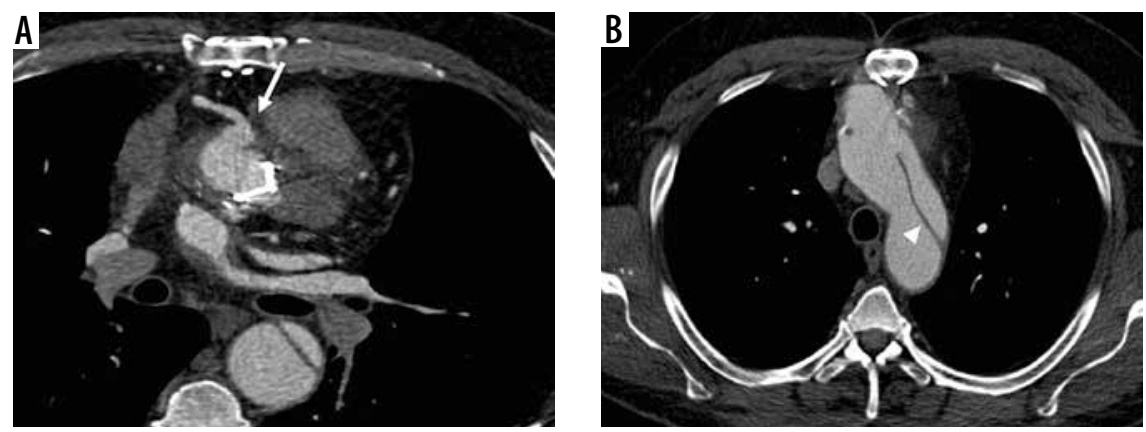

Figure 6. Modified Bentall procedure with aneurysm and distal aortic dissection. Male, 55 years old, with a history of modified Bentall procedure (10 years earlier). Axial enhanced computed tomography scan shows periprosthetic leak with aneurysm of the right coronary ostium ( $A$, arrow), and distal aortic dissection ( $\mathbf{B}$ - intimal flap, arrowhead) with wide false lumen
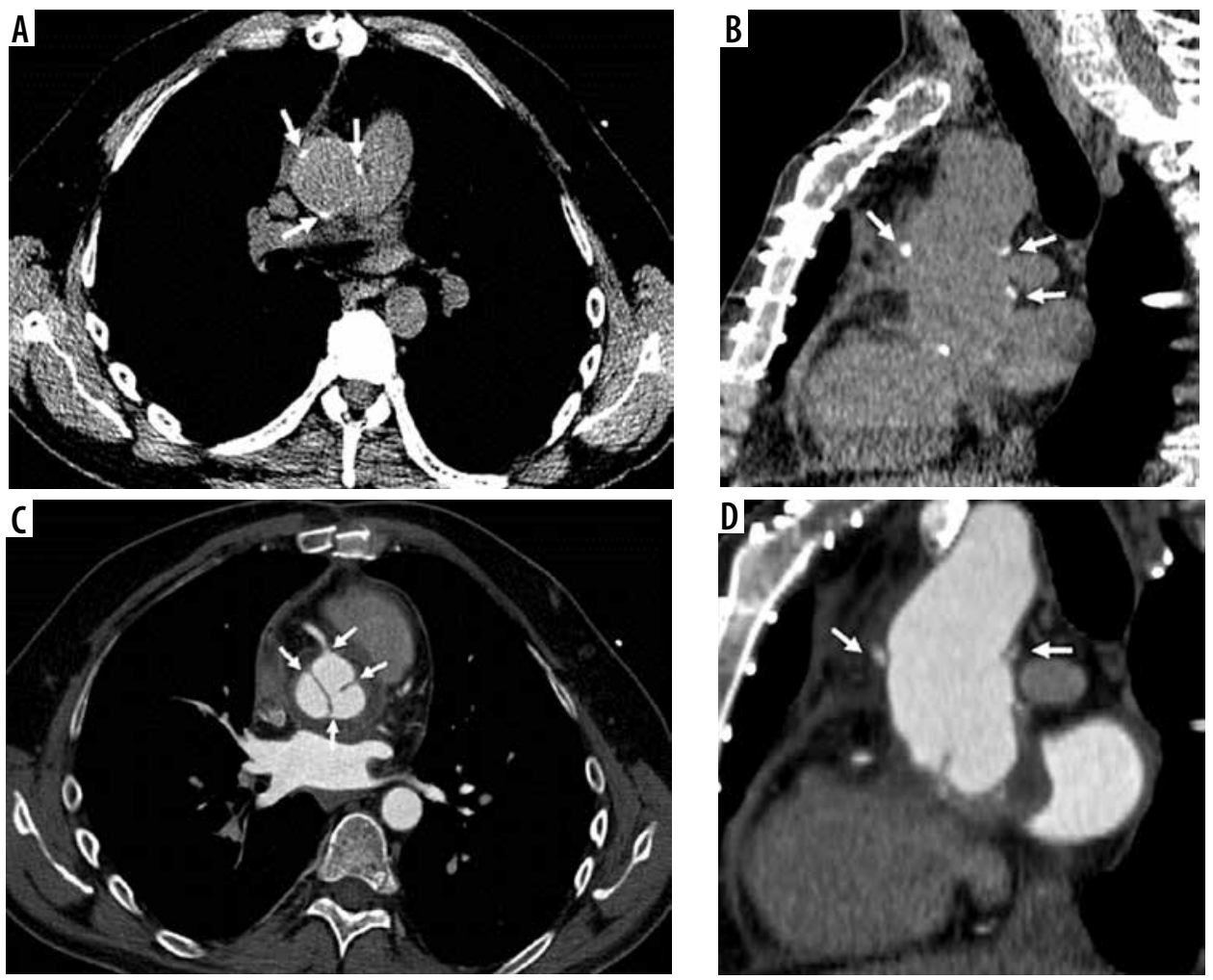

Figure 7. David procedure in Marfan syndrome. Axial and sagittal computed tomography images (A, B - before and C, D - after intravenous contrast administration) show David procedure in a patient with Marfan syndrome. $C$ - shows aortic native valve (arrows)

David- $\mathrm{V}$ technique and allow the patient's valve to be better attached within the artificial sinuses [26].

The main and most important complication of every aortic valve-sparing procedure is aortic valve insufficiency [23].

\section{Arch repair}

In 1983 Borst et al. invented another procedure to repair ascending thoracic aortic aneurysms: the elephant trunk [27].

This procedure consists of two stages; in the first one, a median sternotomy is performed, the aneurysmatic ascending aorta is removed, and an artificial graft is placed within the proximal descending aorta [28]. Three anastomoses are created: a proximal one in the ascending aorta, one with the brachiocephalic vessels, and a distal one after the origin of the left subclavian artery. The distal end of the graft is not connected to any structure and is free to move inside the lumen of the descending aorta.

In the second stage, another graft is placed inside the descending aorta and anastomosed to the previous one through a left thoracoabdominal access [28].

As in the previously mentioned techniques, some variations exist.

In 2003 the 'frozen elephant trunk' technique was introduced. This intervention modifies the second stage of the elephant trunk surgery, where a stent is introduced inside the descending aorta lumen through a femoral artery access, thus removing the need for a second open surgery and lowering mortality rates [29-32] (Figures 8 and 9).

The most common complications are laceration (and rupture) at the distal anastomosis site, air embolism, re- 

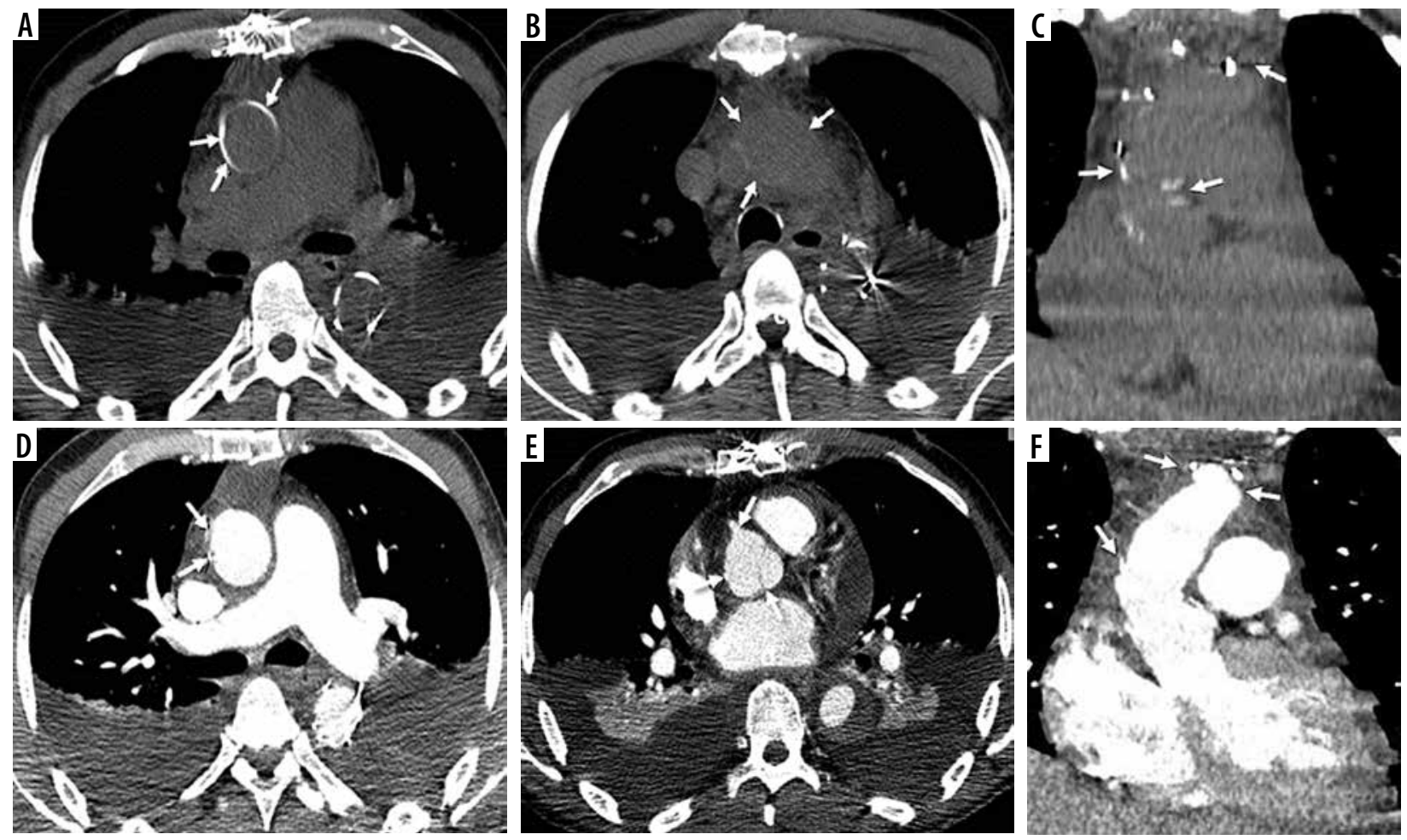

Figure 8. Frozen elephant trunk procedure. Axial and coronal computed tomography images ( $A, B, C$ before and $D, E, F$ after intravenous contrast administration) show frozen elephant trunk procedure
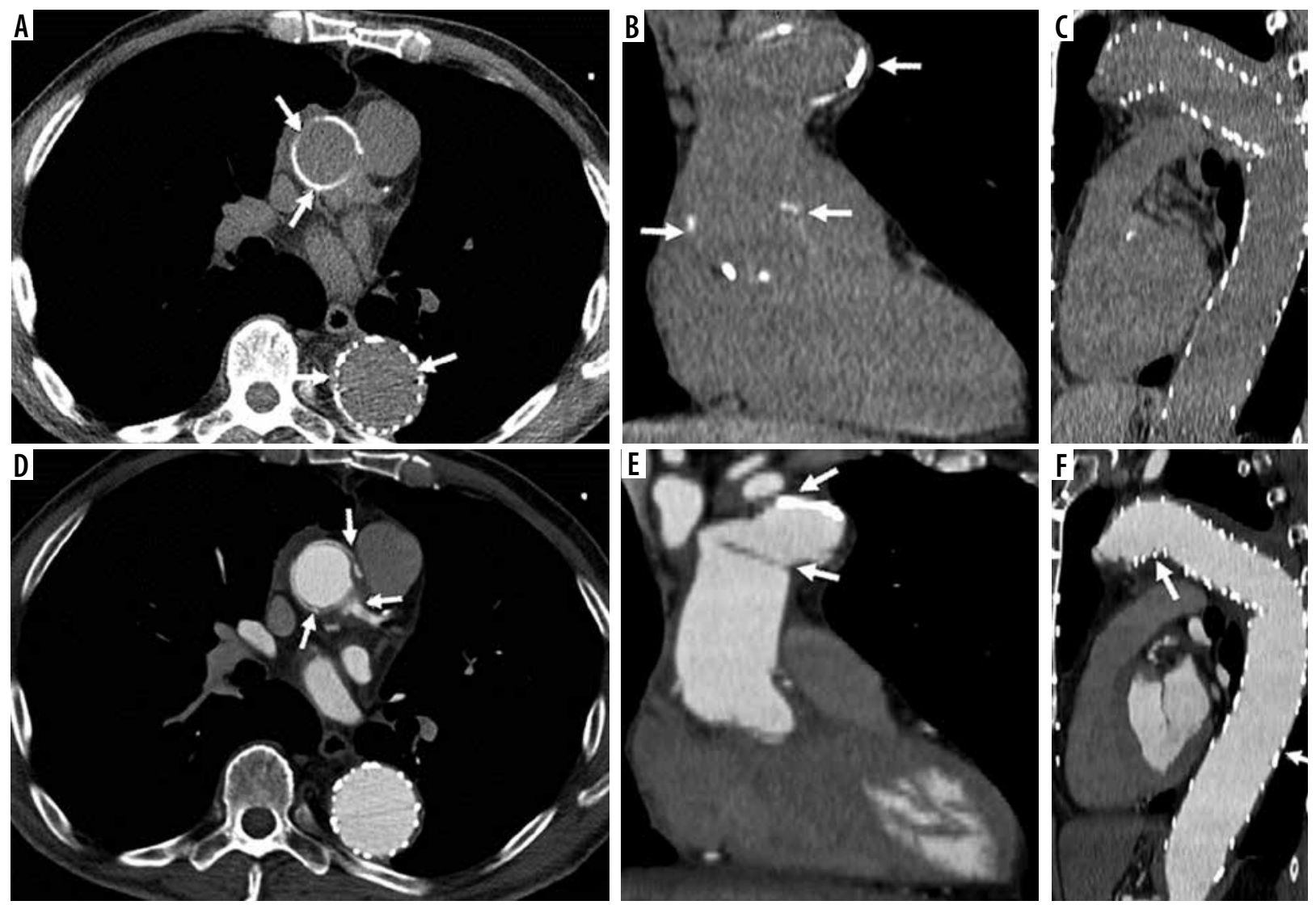

Figure 9. Frozen elephant trunk hybrid procedure. Axial, coronal, and sagittal computed tomography images (A, B, C before and panel D, E, F after intravenous contrast administration) show frozen elephant trunk hybrid procedure. The grafts are well allocated on panel $A$ and $C$, and there are no peri-prosthesis leaks on panel $\mathbf{D}, \mathrm{E}$, and $\mathrm{F}$

current left-sided laryngeal nerve injury, and hence vocal cord paralysis, graft kinking and occlusion, haemorrhage, neurological dysfunction in case of hypoperfusion of the brachiocephalic vessels (such as encephalopathy or stroke), and 

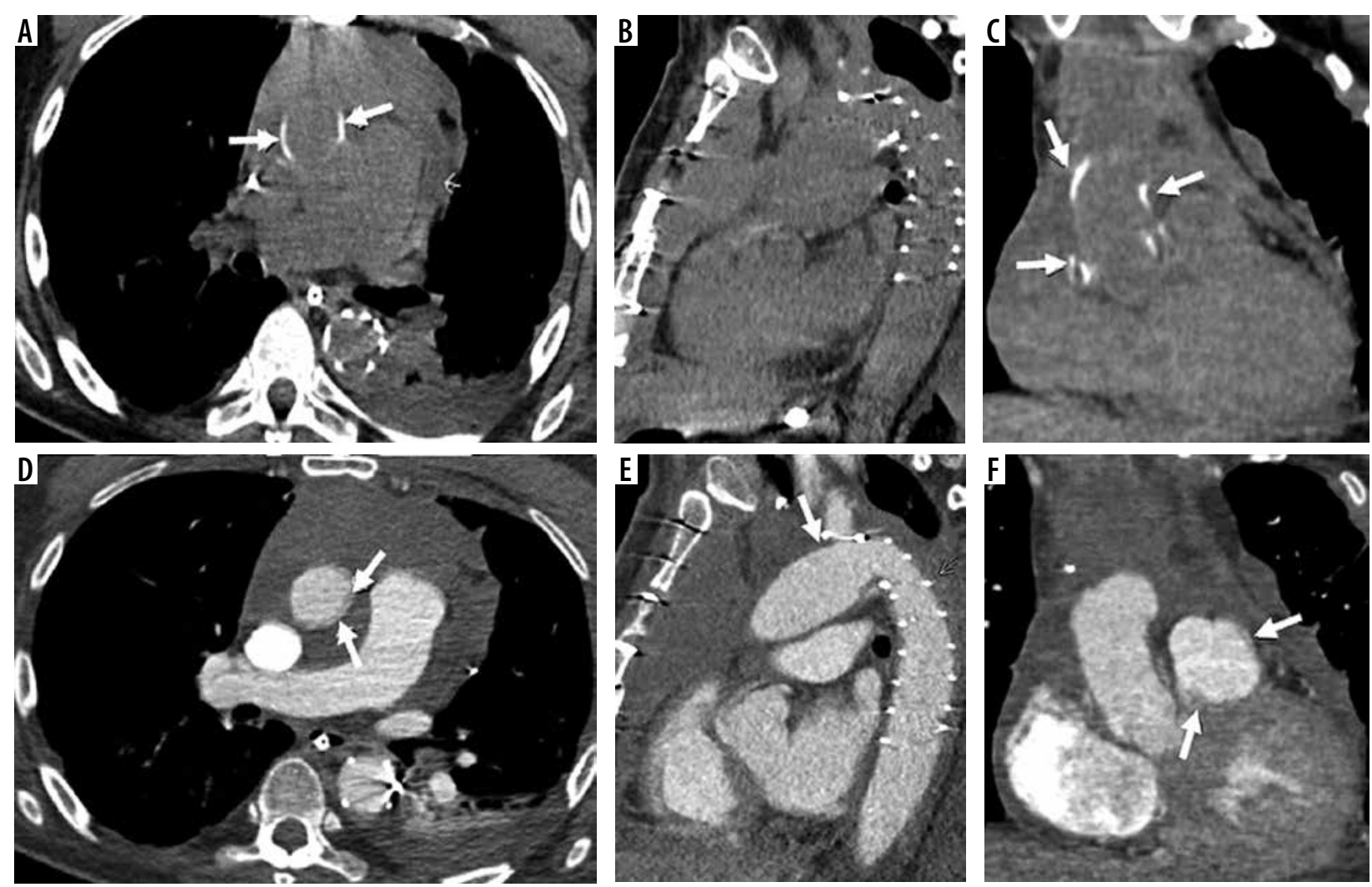

Figure 10. Elephant trunk hybrid procedure with hemopericardium. Axial, sagittal and coronal computed tomography images (A, B, C before and D, E, F after intravenous contrast administration) show elephant trunk hybrid procedure complicated with loculated mediastinal effusion and hemopericardium $(A, D)$

death from rupture of the aneurysm, tamponade, or sepsis [33] (Figure 10).

\section{Conclusions}

A variety of surgical techniques are used to repair a damaged ascending thoracic aorta. It is important for the radiologist to know the type of technique used and to recognize the normal structures of the grafts, in order to be able to spot the imaging features associated with the complications of these procedures.

\section{Conflict of interest}

The authors report no conflict of interest.

\section{References}

1. Gonzalo G, Fernández-Velilla M, Martí M, et al. Endovascular stent-graft treatment of thoracic aortic disease. Radiographics 2005; 25: S229-S244.

2. Agarwal PP, Chughtai A, Matzinger FR, et al. Multidetector CT of thoracic aortic aneurysms. Radiographics 2009; 29: 537-552.

3. Isselbacher EM. Thoracic and abdominal aortic aneurysms. Circulation 2005; 111: 816-828.

4. Elefteriades JA. Natural history of thoracic aortic aneurysms: indications for surgery, and surgical versus nonsurgical risks. Ann Thorac Surg 2002; 74: S1877-S1880; discussion S1892-S1898.

5. Van Hemelrijk C, Renard M, Loeys B. The Loeys-Dietz syndrome: an update for the clinician. Curr Opin Cardiol 2010; 25: 546-551.

6. Svensson LG, Kim KH, Blackstone EH, et al. Bicuspid aortic valve surgery with proactive ascending aorta repair. J Thorac Cardiovasc Surg 2011; 142: 622-629, 629.e1-3.
7. Yamada I, Nakagawa T, Himeno Y, et al. Takayasu arteritis: evaluation of the thoracic aorta with CT angiography. Radiology 1998; 209: 103-109.

8. McMahon MA, Squirrell CA. Multidetector CT of aortic dissection: a pictorial review. Radiographics 2010; 30: 445-460.

9. Williams DM, Lee DY, Hamilton BH, et al. The dissected aorta. III. Anatomy and radiologic diagnosis of branch-vessel compromise. Radiology 1997; 203: 37-44.

10. Patel PD, Arora RA. Pathophysiology, diagnosis, and management of aortic dissection. Ther Adv Cardiovasc Dis 2008; 2: 439-468.

11. Castaner E, Andreu M, Gallardo X, et al. CT in nontraumatic acute thoracic aortic disease: typical and atypical features and complications. Radiographics 2003; 23: S93-S110.

12. Prescott-Focht JA, Martinez-Jimenez S, Hurwitz LM, et al. Ascending thoracic aorta: postoperative imaging evaluation. Radiographics 2013; 33: 73-85. 
13. Hoang JK, Martinez S, Hurwitz LM. MDCT angiography after open thoracic aortic surgery: pearls and pitfalls. AJR Am J Roentgenol 2009; 192: W20-27.

14. Sundaram B, Quint LE, Patel S, et al. CT appearance of thoracic aortic graft complications. AJR Am J Roentgenol 2007; 188: 1273-1277.

15. Wheat MW Jr, Wilson JR, Bartley TD. Successful replacement of the entire ascending aorta and aortic valve. JAMA 1964; 188: 717-719.

16. Bentall H, De Bono A. A technique for complete replacement of the ascending aorta. Thorax 1968; 23: 338-339.

17. Cherry C, DeBord S, Hickey C. The modified Bentall procedure for aortic root replacement. AORN J 2006; 84: 52-55, 58-70.

18. Milano AD, Pratali S, Mecozzi G, et al. Fate of coronary ostial anastomoses after the modified Bentall procedure. Ann Thorac Surg 2003; 75: 1797-1801; discussion 1802.

19. García A, Ferreirós J, Santamaría M, et al. MR angiographic evaluation of complications in surgically treated type A aortic dissection. Radiographics 2006; 26: 981-992.

20. Gelsomino S, Frassani R, Da Col P, et al. A longterm experience with the Cabrol root replacement technique for the management of ascending aortic aneurysms and dissections. Ann Thorac Surg 2003; 75: 126-131.

21. Bowdish ME, Kumar SR, Starnes VA. The Ross procedure: an excellent option in the right hands. Ann Transl Med 2016; 4: 471.

22. Kouchoukos NT, Masetti P, Nickerson NJ, et al. The Ross procedure: long-term clinical and echocardiographic follow-up. Ann Thorac Surg 2004; 78: 773-781; discussion 773-781.

23. David TE, Ivanov J, Armstrong $S$, et al. Aortic valve-sparing operations in patients with aneurysms of the aortic root or ascending aorta. Ann Thorac Surg 2002; 74: S1758-S1761; discussion S1792-S1799.

24. Sarsam MA, Yacoub M. Remodeling of the aortic valve anulus. J Thorac Cardiovasc Surg 1993; 105: 435-438.
25. David TE, Feindel CM. An aortic valve-sparing operation for patients with aortic incompetence and aneurysm of the ascending aorta. J Thorac Cardiovasc Surg 1992; 103: 617-621; discussion 622.

26. Demers P, Miller DC. Simple modification of “T. David-V" valve-sparing aortic root replacement to create graft pseudosinuses. Ann Thorac Surg 2004; 78: 1479-1481.

27. Borst H, Walterbusch G, Schaps D. Extensive aortic replacement using "elephant trunk" prosthesis. Thorac Cardiovasc Surg 1983; 31: 37-40.

28. LeMaire SA, Carter SA, Coselli JS. The elephant trunk technique for staged repair of complex aneurysms of the entire thoracic aorta. Ann Thorac Surg 2006; 81: 1561-1569; discussion 1569.

29. De Paulis R, Di Bartolomeo R, Murana G, et al. Frozen versus conventional elephant trunk technique: application in clinical practice. Eur J Cardiothorac Surg 2017; 51 (suppl 1): i20-i28.

30. Karck M, Chavan A, Hagl C, et al. The frozen elephant trunk technique: a new treatment for thoracic aortic aneurysms. J Thorac Cardiovasc Surg 2003; 125: 1550-1553.

31. Ma WG, Zheng J, Sun LZ, et al. Open stented grafts for frozen elephant trunk technique: technical aspects and current outcomes. Aorta (Stamford) $2015 ; 3: 122-135$.

32. Sun L, Qi R, Zhu J, et al. Total arch replacement combined with stented elephant trunk implantation: a new "standard" therapy for type a dissection involving repair of the aortic arch? Circulation 2011; 123: 971-978.

33. Johnson PT, Corl FM, Black JH, Fishman EK. The elephant trunk procedure for aortic aneurysm repair: an illustrated guide to surgical technique with CT correlation. AJR Am J Roentgenol 2011; 197: W1052-1059. 